\section{Small bowel perforation caused by an impacted endocapsule}
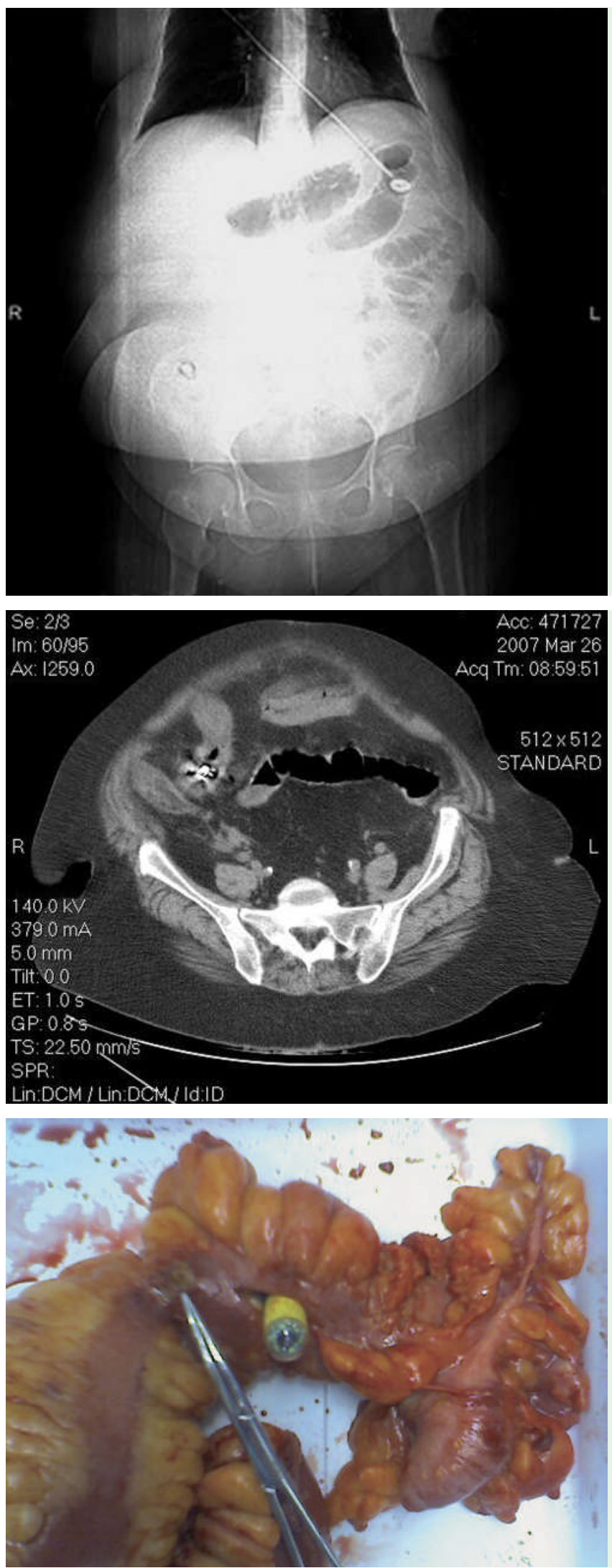

Fig. 1 Metallic foreign body in the region of the terminal ileum.

Fig. 2 Intraluminal metallic foreign body with inflammatory changes noted surrounding the terminal ileum.

Fig. 3 The tip of the Kelly clamp points to the area of perforation. The endocapsule is taken out of the surgical incision in the terminal ileum after the resection.
Capsule endoscopy has become a common diagnostic study to evaluate smallbowel lesions. One of its major complications is small-bowel obstruction due to capsule impaction [1]. So far, there have been two documented cases of smallbowel perforation caused by the impacted capsule [2,3]. We report the case of a 75-year-old woman with Crohn's disease who presented with acute abdominal pain and vomiting. CT of the abdomen and pelvis showed small-bowel obstruction with a metallic foreign body in the distal ileum ( $\bullet$ Fig. 1 and 2). Seventeen days earlier, the patient had undergone capsule endoscopy for evaluation of persistent diarrhea related to Crohn's disease, which revealed multiple strictures in the small-bowel.

Exploratory laparotomy revealed smallbowel dilation leading to the obstruction near the terminal ileum. Ischemic changes were noted throughout the terminal ileum consistent with Crohn's disease. In the same region, a 3-mm perforation and a retained endocapsule were found ( Fig. 3). This section of the small bowel was resected and a side-to-side anastomosis was created.

Capsule impaction occurs when an endocapsule remains in the digestive tract for more than 2 weeks [3]. Patients with Crohn's disease are at high risk of capsule impaction due to their tendency to form strictures. In our case, the location of the impaction/perforation and the length of retention time strongly suggest that the perforation was caused by the retained endocapsule. Studies on capsule impaction and/or small-bowel obstruction show incidence rate of $0.75-21 \%[3,4]$. However, this rate may not be typical of capsule endoscopy in general, since the studies state that most participating patients undergo imaging tests prior to capsule endoscopy to rule out small-bowel strictures [4], whereas patients not enrolled in studies may not receive a complete set of evaluations prior to capsule endoscopy or may not always attend for follow-up, and both these factors could increase the rate of capsule impaction. In order to prevent such complications, all patients with suspected Crohn's disease must undergo imaging studies such as CT scan and upper gastrointestinal series/ small bowel follow through to exclude strictures before they undergo capsule endoscopy. Providers must have a high index of suspicion of capsule impaction in patients who do not excrete the endocapsule within a reasonable amount of 
time and surgical intervention should be planned.

Endoscopy_UCTN_Code_CPL_1AI_2AB

S. Um ${ }^{1}$, H. Poblete' ${ }^{1}$, J. Zavotsky ${ }^{2}$

1 Hahnemann University Hospital, Philadelphia, Pennsylvania, USA

2 St. Peter's University Hospital, New Brunswick, New Jersey, USA

\section{References}

1 Barkin JS, Friedman S. Wireless capsule endoscopy requiring surgical intervention: the world's experience. Am J Gastroenterol 2002; 97: S298

2 Gonzalez Carro P, Picazo Yuste J, Fernández Díez $S$ et al. Intestinal perforation due to retained capsule endoscope. Endoscopy 2005; 37: 684

3 Lin OS, Otto S et al. Acute symptomatic small bowel obstruction due to capsule impaction. Gastrointest Endosc 2007; 65: $725-$ 728

4 Cheifetz AS, Kornbluth AA, Legnani $P$ et al. The risk of retention of the capsule endoscope in patients with known or suspected Crohn's disease. Am J Gastroenterol 2006; 101: $2218-2222$

5 Gaba RC, Schlesinger PK, Wilbur AC. Endoscopic video capsule: radiologic findings of spontaneous entrapment in small intestinal diverticula. AJR Am J Roentgenol 2005; 185: 1048
Bibliography

DOI 10.1055/s-2007-995694

Endoscopy 2008; 40: E122-E123

(c) Georg Thieme Verlag KG Stuttgart · New York . ISSN 0013-726X

Corresponding author

\section{S. Um, MD}

Department of Surgery

Hahnemann University Hospital

Broad and Vine St

Philadelphia

PA 19102

USA

scott_um@hotmail.com 